\title{
The Anaesthetic Biobank of Cerebrospinal fluid: a unique repository for neuroscientific biomarker research
}

\author{
Celien Tigchelaar ${ }^{1} \wedge$, Sawal D. Atmosoerodjo ${ }^{1}$, Martijn van Faassen ${ }^{2}$, Klaas J. Wardenaar ${ }^{3}$, \\ Peter P. De Deyn ${ }^{4,5,6,7}$, Robert A. Schoevers ${ }^{3} \wedge$, Ido P. Kema ${ }^{2} \wedge$, Anthony R. Absalom ${ }^{1} \wedge$ \\ ${ }^{1}$ Department of Anesthesiology, University Medical Center Groningen, University of Groningen, Groningen, The Netherlands; ${ }^{2}$ Department of \\ Laboratory Medicine, University Medical Center Groningen, University of Groningen, Groningen, The Netherlands; ${ }^{3}$ Department of Psychiatry, \\ University Medical Center Groningen, University of Groningen, Groningen, The Netherlands; ${ }^{4}$ Department of Neurology and Alzheimer Center, \\ University Medical Center Groningen, University of Groningen, Groningen, The Netherlands; ${ }^{5}$ Laboratory of Neurochemistry and Behavior, \\ Department of Biomedical Sciences, Institute Born-Bunge, University of Antwerp, Belgium; ${ }^{6}$ Department of Neurology and Memory Clinic, \\ Hospital Network Antwerp (ZNA) Middelheim and Hoge Beuken, Antwerp, Belgium; ${ }^{7}$ Biobank, Institute Born-Bunge, University of Antwerp, \\ Antwerp, Belgium \\ Contributions: (I) Conception and design: All authors; (II) Administrative support: C Tigchelaar, SD Atmosoerodjo; (III) Provision of study materials \\ or patients: C Tigchelaar, SD Atmosoerodjo, M van Faassen, IP Kema, AR Absalom; (IV) Collection and assembly of data: C Tigchelaar, SD \\ Atmosoerodjo, M van Faassen; (V) Data analysis and interpretation: C Tigchelaar, KJ Wardenaar, AR Absalom; (VI) Manuscript writing: All authors; \\ (VII) Final approval of manuscript: All authors. \\ Correspondence to: Celien Tigchelaar. Department of Anesthesiology, University Medical Center Groningen, University of Groningen, Hanzeplein 1, \\ 9713 GZ Groningen, The Netherlands. Email: c.tigchelaar@umcg.nl.
}

Background: The pathophysiology of numerous central nervous system disorders remains poorly understood. Biomarker research using cerebrospinal fluid (CSF) is a promising way to illuminate the neurobiology of neuropsychiatric disorders. CSF biomarker studies performed so far generally included patients with neurodegenerative diseases without an adequate control group. The Anaesthetic Biobank of Cerebrospinal fluid (ABC) was established to address this. The aims are to (I) provide healthy-control reference values for CSF-based biomarkers, and (II) to investigate associations between CSF-based candidate biomarkers and neuropsychiatric symptoms.

Methods: In this cross-sectional study, we collect and store CSF and blood from adult patients undergoing spinal anaesthesia for elective surgery. Blood $(20.5 \mathrm{~mL})$ is collected during intravenous cannulation and CSF $(10 \mathrm{~mL})$ is aspirated prior to intrathecal local anaesthetic injection. A portion of the blood and CSF is sent for routine laboratory analyses, the remaining material is stored at $-80{ }^{\circ} \mathrm{C}$. Relevant clinical, surgical and anaesthetic data are registered. A neurological examination and Montreal Cognitive Assessment (MoCA) are performed pre-operatively and a subset of patients fill in questionnaires on somatic and mental health (depression, anxiety and stress).

Results: Four-hundred-fifty patients (58\% male; median age: 56 years) have been enrolled in the ABC. The planned spinal anaesthetic procedure was not attempted for various reasons in eleven patients, in fourteen patients the spinal puncture failed and in twelve patients CSF aspiration was unsuccessful. A mean of $9.3 \mathrm{~mL} \mathrm{CSF}$ was obtained in the remaining 413 of patients. Most patients had a minor medical history and $60 \%$ scored in the normal range on the MoCA (median score: 26).

Conclusions: The ABC is an ongoing biobanking project that can contribute to CSF-based biomarker research. The large sample size with constant sampling methods and extensive patient phenotyping provide excellent conditions for future neuroscientific research.

\footnotetext{
$\wedge$ ORCID: Celien Tigchelaar, 0000-0002-7505-8827; Martijn van Faassen, 0000-0002-9142-6263; Klaas J. Wardenaar, 0000-0003-1025615X; Peter P. De Deyn, 0000-0002-2228-2964; Robert A. Schoevers, 0000-0003-0760-9866; Ido P. Kema, 0000-0003-1166-6169; Anthony R. Absalom, 0000-0001-7563-9157.
} 
Keywords: Biomarkers; cerebrospinal fluid (CSF); central nervous system; mental disorders; neurosciences

Submitted Jun 04, 2020. Accepted for publication Dec 18, 2020.

doi: $10.21037 / \mathrm{atm}-20-4498$

View this article at: http://dx.doi.org/10.21037/atm-20-4498

\section{Introduction}

Biomarker research using cerebrospinal fluid (CSF) is a promising way to improve our knowledge of the neurobiology and increase the accuracy of the diagnostic process and treatment of numerous central nervous system (CNS) disorders. Neuropsychiatric disorders are very common and have significant socio-economic consequences (1-3). These diseases are commonly identified by clusters of symptoms (i.e., syndromes), which are often very heterogeneous, have a variable course and respond inconsistently to treatment $(4,5)$. Therefore, there is an urgent need for a better understanding of the underlying pathological processes. Fortunately, advances in imaging and laboratory techniques over the last decades have led to a shift from identifying neuropsychiatric diseases purely based on clinical definitions, to identifying them based on neurobiological mechanisms $(6,7)$. A considerable part of research into these mechanisms focuses on the investigation of biomarkers (8). Novel biomarkers could serve to improve screening and/or diagnostic procedures by facilitating early detection, and provide accurate on prognosis and response to treatment. Biomarkers might also act as targets for new treatments (7-9). These biomarkers can be measured in blood, saliva and/or urine, but for neurological and psychiatric disorders, the study of biomarkers in CSF is especially informative, given the proximity of CSF to the CNS.

For a long time, the main source of information about the CSF was from analysis of CSF from patients with suspected bacterial meningitis. More recent work has progressed beyond the traditional biochemical and microbiological analyses in these patients. A recent example is a study that investigated the role of the tryptophankynurenine-nicotinamide adenine dinucleotide pathway in patients with acute CNS infections (bacterial and viral), but then compared the findings with those from patients with auto-immune encephalitis, multiple sclerosis, and patients with non-inflammatory CNS conditions who cannot be considered to be neurologically normal (10).

In recent decades it has become apparent that changes in
CSF composition may hold clues about the pathophysiology of neurodegenerative disorders, such as Parkinson's disease (PD) (11), multiple sclerosis (MS) (12) and Frontotemporal Dementia (13). Therefore, for patients with these suspected neurological disorders, a lumbar puncture (LP) for CSF collection is often performed for diagnostic purposes $(14,15)$. This is however mostly informed by knowledge of reference values in patients suspected of, or known to have, these disorders (16-18). For psychiatric disorders such as major depressive disorder, biomarker research has mostly involved plasma rather than CSF analysis $(8,19,20)$ and the few studies investigating CSF biochemistry have frequently produced inconsistent results $(21,22)$.

Most CSF biomarker studies have included patients with neurological symptoms as reference (in retrospect after excluding a CNS disease), have used very small control groups or included patients undergoing spinal anaesthesia (9,10,18,23-27). Even studies designed to establish reference ranges for CSF neurotransmitters included symptomatic patients $(28,29)$ or consisted of a small cohort with healthy volunteers (30). To assist with interpretation of new findings, investigators ultimately depend on the availability of sufficiently large databases containing the results of assays of biomaterials (CSF, blood and urine), and concomitantly assessed clinical features and personal characteristics, of a range of patients and healthy controls. Due to the attendant risks of a LP, such as infection, neurological injury and post-dural puncture headache (PDPH) (31), it is difficult to obtain CSF from healthy controls because of the ethical considerations involved $(9,27)$.

A second limitation of previous studies in CNS diseases has been these studies' small sample sizes that limit statistical power to detect small but potentially relevant biological effects $(16,32)$. One way to address this, is to combine smaller cohorts with similar designs. However, most cohorts have a strong variation in sampling, storing and analysis methods which makes it hard to draw final conclusions. Another approach to address the problem of a small sample size is by setting up large CSF biobanks. Most initiatives of CSF biobanking focus on neurodegenerative diseases like AD (23,33-35), Parkinson disease (33), MS (36), 
and amyotrophic lateral sclerosis (ALS) (35). Despite these initiatives, even in these large biobanks the number of available healthy controls is often small. Biobanking studies in the field of mental disorders mainly collect biomaterials like plasma, saliva and urine for genetic research $(37,38)$, since a LP is not part of the routine diagnostic procedure in psychiatry.

Given the promise of CSF biobanking for many neurobiological research areas and the abovementioned limitations in existing sampling frameworks, the Anaesthetic Biobank of Cerebrospinal fluid (ABC) was started. The $\mathrm{ABC}$ was established by the Anesthesiology and Clinical Chemistry departments of the University Medical Center Groningen (UMCG) in October 2016. CSF and blood are collected from patients undergoing planned spinal anaesthesia for elective surgery, a relatively neurological healthy population. Spinal anaesthesia requires a LP to enable intrathecal administration of a local anaesthetic agent. We are thus able to collect CSF from these relatively healthy surgical patients, without significant risks over and above the usual risks of an LP. Aspiration of up to $30 \mathrm{~mL}$ of CSF has been shown to be well-tolerated and safe (39). As we only planned to aspirate $10 \mathrm{~mL}$ of CSF, we did not consider that this would significantly increase the risk of PDPH (the most common serious complication of spinal puncture) $(31,39)$. In addition, we capture useful clinical information that is routinely collected before and during the surgical procedures, perform simple neurological and cognitive function assessments during their hospital admission with little additional inconvenience to the patients. The purpose of our study is to contribute towards a better understanding of the neurophysiology and to identify CSF-based biomarkers for a range of neuropsychiatric disorders.

The objective of this paper is to describe the methods, initial results and characteristics of the study population. By these means, our aim is to show an example of CSF biobanking in clinical practice, and to suggest how the collected data might be applied to multiple fields of interest. We present the following article in accordance with the STROBE reporting checklist (available at http://dx.doi. org/10.21037/atm-20-4498).

\section{Methods}

\section{Study design and etbical considerations}

In this cross-sectional study, CSF, blood and clinical data are collected from patients undergoing spinal anaesthesia for elective surgical procedures at the University Medical Center Groningen (UMCG). The ABC and study procedures reported here were approved by the Medical Ethical Committee of the UMCG (registration number 2016-174). Written informed consent is obtained from all participants for collection and storage of material and medical data to conduct research consistent with the aforementioned purposes of the $\mathrm{ABC}$. The additional burden and risks for participants is considered minimal because spinal puncture is part of standard care. The $\mathrm{ABC}$ procedures are designed to be consistent with the recommendations of an international consensus protocol for the standardization of CSF collection and biobanking (16).

\section{Patients}

All patients 18 years of age and older scheduled for elective surgery under spinal anaesthesia are invited to participate, except for patients scheduled for caesarean section. Patients planned for spinal anaesthesia are identified using the preoperative anaesthesiological screening.

\section{Lumbar puncture and spinal anaesthetic procedure}

Spinal anaesthesia is conducted using a standard protocol which mandates sterile procedure and use of a non-cutting lumbar puncture needle. Choices such as patient position, inter-vertebral space, needle gauge and type and dose of intrathecal local anaesthetic are at the discretion of the anaesthesiologist responsible for the clinical care of the patient. After the anaesthesiologist reaches the intrathecal space, CSF is aspirated via the spinal needle into five $2 \mathrm{~mL}$ syringes. The local anaesthetic agent is then administered intrathecally. After 10 minutes, sensory block height is assessed with ice to determine the extent of loss of temperature sensation.

\section{CSF and blood acquisition, storage and analysis}

\section{CSF}

CSF is acquired as described above. The first $2 \mathrm{~mL}$ of CSF is used for routine analyses (albumin, total protein, glucose, leucocyte and erythrocyte count). The remaining four $2 \mathrm{~mL}$ CSF fractions are transferred into $2 \mathrm{~mL}$ ordered tubes wrapped in aluminium foil and directly put on ice. We aim to transport samples to the laboratory within 20 minutes after CSF collection with a maximum of two 
hours until freezing. CSF is centrifuged at $1,000 \times \mathrm{g}$ for 10 minutes at $4{ }^{\circ} \mathrm{C}$. Every $2 \mathrm{~mL}$ fraction is divided into four aliquots of $500 \mu \mathrm{L}$ and the order is registered. Glutathione and dithiothreitol (DTT) are added to one of the aliquots of the fourth and fifth fraction. A total of sixteen aliquots are stored at $-80^{\circ} \mathrm{C}$.

\section{Blood}

Prior to the lumbar puncture, during intravenous cannulation, $20.5 \mathrm{~mL}$ of blood is collected. $10.5 \mathrm{~mL}$ of this blood is collected for routine analyses into a $4.5 \mathrm{~mL}$ lithium heparin tube (albumin and total protein), a $4 \mathrm{~mL}$ ethylenediaminetetraacetic acid (EDTA) tube (leucocyte count) and a $2 \mathrm{~mL}$ sodium fluoride tube (glucose). The remaining blood is collected into a $10 \mathrm{~mL}$ EDTA tube, immediately put on ice and transported to the laboratory where cells are removed from plasma by centrifugation at 2,000 $\times \mathrm{g}$ for 10 minutes at $4{ }^{\circ} \mathrm{C}$. Plasma is then divided into ten aliquots of $500 \mu \mathrm{L}$, of which two aliquots contain glutathione and two aliquots DTT for stabilisation. The ten aliquots are stored at $-80^{\circ} \mathrm{C}$ with a maximum time delay of four hours between sample collection and storage (the target delay is 2 hours).

\section{Clinical and other data collection, processing and storage}

Data are collected according to standard operating procedures (SOPs) specifically defined for the ABC and registered in the (electronic) case report form. To minimize the privacy risk for participants and to ensure data integrity and confidentially, a unique study number is assigned to each participant (coding), and access to the data is restricted.

\section{Patient characteristics}

Data regarding patient demographics, medical history, allergies, intoxications and medication use are collected. Demographic variables include age, gender, and ethnicity. Weight, height, blood pressure, and heart rate measured at the preoperative anaesthesiology screening, and the American Society of Anesthesiologists (ASA) score are noted. Data is collected on medical conditions (diagnosis and date) and categorized by one of the two coordinating investigators of the $\mathrm{ABC}$ into the following categories: general, cardiovascular, respiratory, gastrointestinal, neurological, endocrinological, urological, locomotor, haematological, and psychiatric medical history. Additionally, data on alcohol consumption, smoking behaviour, drug use habits, and current and past-year medication use are collected.

\section{Physical examination and cognitive assessment}

Preoperatively a screening neurological examination and the Montreal Cognitive Assessment (MoCA) (40) are performed if logistically possible. For the neurological examination a checklist consisting of 26 items (see Figure S1) was followed to test for cranial nerve function, sensory and motor function, coordination, gait, and deep tendon reflexes. Abnormal findings are described with free text as accurately as is possible. The examination is regarded as normal if all items for that aspect are tested as normal.

\section{Psychosomatic questionnaires}

Between November 2017 and October 2019, patients were asked to fill in a daily diary starting seven days prior to surgery until the day of surgery. The diary consists of the following previously validated questionnaires: the 9-item Patient Health Questionnaire (PHQ) for depression, 15-item PHQ for somatization, 7-item General Anxiety Disorder (GAD) for anxiety symptoms (41), and four additional questions about perception of stress. From June 2019 onwards, the Inventory of Depressive Symptoms - Self Report (IDS-SR) is used to assess depressive symptoms (42). The IDS-SR is completed 2 weeks before (baseline) and 2 weeks after surgery (follow-up).

\section{Surgical and spinal anaesthesia procedure}

Date and type of surgical procedure and the use of premedication (medication administered prior to CSF aspiration) are registered. Details of the spinal anaesthesia and CSF collection are recorded. Characteristics of the spinal technique that are recorded include patient position during the procedure, vertebral puncture level, needle type and size, presence of paraesthesia, and anaesthetic type, concentration and volume. Sensory block height, total CSF volume aspirated, time taken to aspirate CSF and presence/ absence of blood in CSF is recorded. Hemodynamic parameters (blood pressure and heart rate) at the start of CSF aspiration are registered. Post-operative length-ofstay and complications such as an incomplete spinal block, vasovagal reaction and PDPH are monitored and recorded.

\section{Sample collection and processing}

Time of sample collection and storage is registered and any deviations from the SOPs regarding transport, processing 


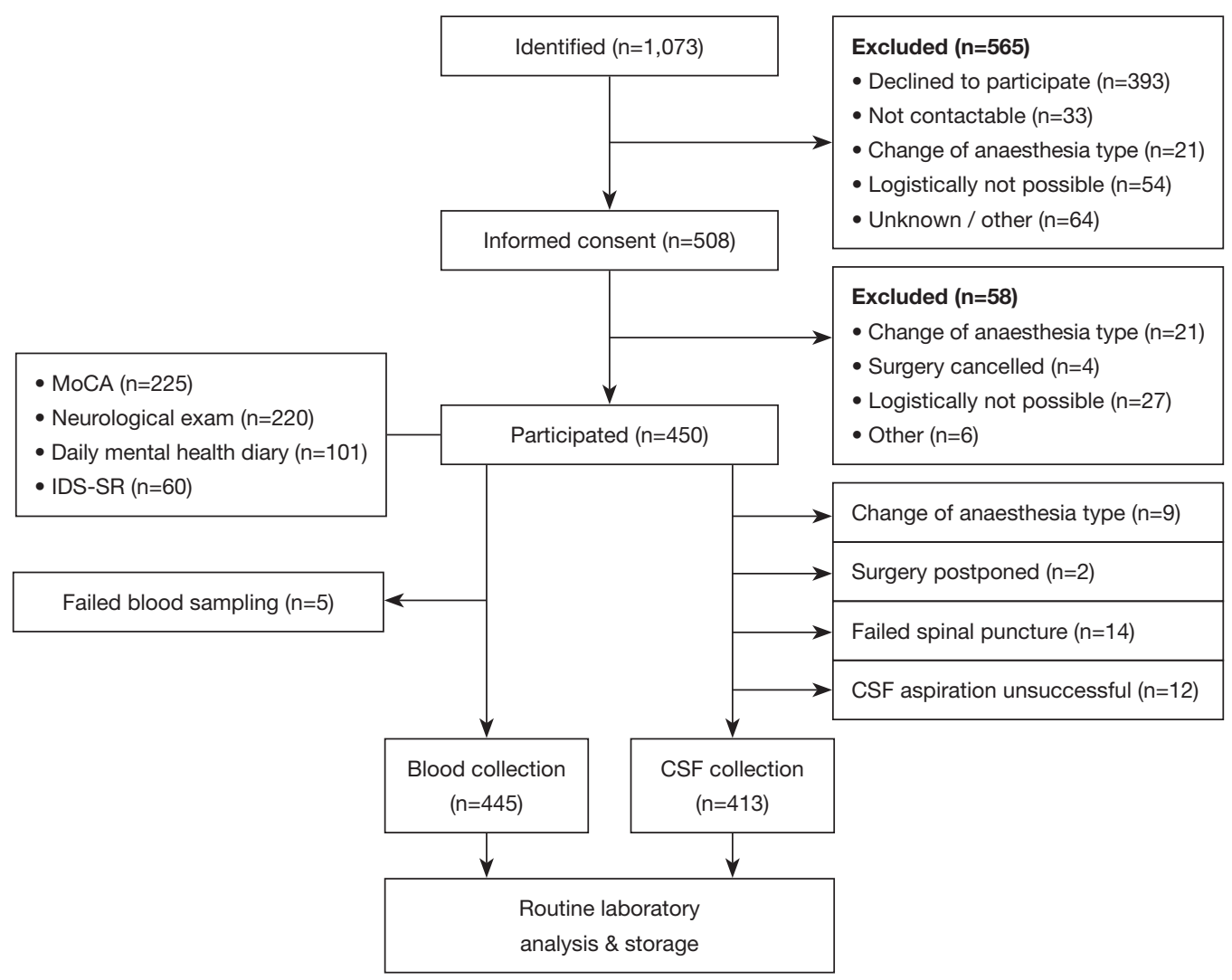

Figure 1 Study flow diagram of patient inclusion. CSF, cerebrospinal fluid; MoCA, Montreal Cognitive Assessment; IDS-SR, Inventory of Depressive Symptoms-Self Report.

and storage of biomaterials are documented.

\section{Statistical analysis}

Analyses were performed using SPSS statistics software (v23) and encompassed mainly descriptive statistics. Normally distributed continuous data are presented as mean \pm standard deviation (SD) and non-normally distributed continuous data with the median [inter quartile range (IQR)]. Discrete data are expressed by category frequencies and percentages.

\section{Results}

We report here the data on the 450 patients enrolled in the study between October 2016 and March 2020. Patient enrolment is summarised in Figure 1. We identified and approached 1,073 patients of whom 508 patients gave informed consent, 393 patients declined to participate and 172 were excluded for other reasons. Most patients that declined did so because of concerns about the anticipated additional burden participation would cause.

Patient characteristics are shown in Table 1. Of patients enrolled, $58 \%$ were male and the median age was 56 years (IQR: 37-67). Body mass index (BMI) ranged from 18 to 51 with a median of $27 \mathrm{~kg} / \mathrm{m}^{2}$. Almost half of patients were ASA status I and were discharged home on the day of surgery (47\%). Of the enrolled patients 68 (16\%) currently smoked tobacco, 279 (68\%) used alcohol and 15 (4\%) used recreational drugs. The majority of patients underwent orthopaedic surgery (63\%).

\section{Spinal procedure}

In eleven patients, after blood had been collected, for various reasons the planned spinal anaesthetic procedure was not attempted. The details of the attempted spinal anaesthetic technique in the remaining 439 patients are listed in Table 2. In fourteen patients, spinal puncture failed despite several attempts, leaving 425 patients, in whom the 
Table 1 Patient characteristics

\begin{tabular}{|c|c|}
\hline Characteristics & Value \\
\hline $\mathrm{N}$ & 450 \\
\hline \multicolumn{2}{|l|}{ Demographics } \\
\hline Gender, male/female & $261[58] / 189$ [42] \\
\hline Age (years) & 56 [37-67]; range 18-92 \\
\hline \multicolumn{2}{|l|}{ Age categorical (years) } \\
\hline $18-19$ & $13[3]$ \\
\hline 20-39 & $112[25]$ \\
\hline $40-59$ & $144[32]$ \\
\hline $60-79$ & $160[35]$ \\
\hline $80-99$ & $21[5]$ \\
\hline \multicolumn{2}{|l|}{ Ethnicity } \\
\hline Caucasian & $443[98.4]$ \\
\hline African & $4[0.9]$ \\
\hline Mixed & $2[0.4]$ \\
\hline Other & $1[0.2]$ \\
\hline BMI $\left(k g / m^{2}\right)$ & 27 [24-31]; range 18-51 \\
\hline \multicolumn{2}{|l|}{ ASA classification } \\
\hline ASA I & $203[45]$ \\
\hline ASA II & $198[44]$ \\
\hline ASA III & $48[11]$ \\
\hline ASA IV & $1[0.2]$ \\
\hline \multicolumn{2}{|l|}{ Lifestyle factors } \\
\hline \multicolumn{2}{|l|}{ Smoking $(n=423)$} \\
\hline Never & $262[62]$ \\
\hline Current & $68[16]$ \\
\hline Former & 93 [22] \\
\hline Alcohol use, yes/no $(n=408)$ & 279 [68]/129 [32] \\
\hline Drug use, yes/no $(n=415)$ & $15[4] / 400[96]$ \\
\hline \multicolumn{2}{|l|}{ Surgery type } \\
\hline Orthopaedic & 283 [63] \\
\hline Urological & 93 [21] \\
\hline Surgical & $47[10]$ \\
\hline Gynaecological & $27[6]$ \\
\hline
\end{tabular}

Data are presented as mean $\pm \mathrm{SD}$, median [IQR] or frequency [\%]. BMI, body mass index; ASA, American Society of Anesthesiologists.
Table 2 Spinal technique characteristics

\begin{tabular}{|c|c|}
\hline Characteristics & Value \\
\hline $\mathrm{N}$ & 439 \\
\hline Patient position (seated/lateral) $(n=437)$ & $414[95] / 23[5]$ \\
\hline \multicolumn{2}{|l|}{ Puncture position (spinal level) $(n=426)$} \\
\hline L1-L2 & $5[1]$ \\
\hline L2-L3 & $66[16]$ \\
\hline L3-L4 & $302[71]$ \\
\hline L4-L5 & $53[12]$ \\
\hline \multicolumn{2}{|l|}{ Local anaesthetic agent $(n=424)$} \\
\hline Bupivacaine isobaric $0.5 \%$ & $57[13]$ \\
\hline Bupivacaine hyperbaric $0.5 \%$ & 138 [33] \\
\hline Prilocaine hyperbaric $2 \%$ & $229[54]$ \\
\hline Sensory block height (dermatome) $(n=409)$ & $\begin{array}{l}\text { T8 [T6-T10]; } \\
\text { range C4-L5 }\end{array}$ \\
\hline Bupivacaine isobaric $0.5 \%(n=56)$ & $\begin{array}{l}\text { T6 [T4-T10]; } \\
\text { range C6-L1 }\end{array}$ \\
\hline Bupivacaine hyperbaric $0.5 \%(n=135)$ & $\begin{array}{l}\text { T8 [T6-T10]; } \\
\text { range T1-L4 }\end{array}$ \\
\hline Prilocaine hyperbaric $2 \%(n=217)$ & $\begin{array}{l}\text { T9 [T6-T10]; } \\
\text { range C4-L5 }\end{array}$ \\
\hline \multicolumn{2}{|l|}{ Complications } \\
\hline Failed spinal anaesthesia & $14[3]$ \\
\hline Inadequate block & $15[3]$ \\
\hline Vasovagal response & $30[7]$ \\
\hline Post dural puncture headache & $3[0.7]$ \\
\hline
\end{tabular}

Data are presented as mean $\pm \mathrm{SD}$, median [IQR] or frequency [\%].

intrathecal space was reached and identified.

The spinal puncture was mostly performed with the patient in the seated position (95\%) and in all cases, a 'pencil point' (atraumatic) 25 Gauge spinal needle was used. Nine patients received a combined spinal-epidural procedure for post-operative analgesia. Hyperbaric prilocaine was the most commonly used local anaesthetic (54\%). The median local anaesthetic volume injected intrathecally was $3.0 \mathrm{~mL}$ for all three anaesthetic agents (range, 1.0-4.5 mL).

The median sensory block height 10 minutes after spinal injection was dermatome $\mathrm{T} 8$, with a range from $\mathrm{C} 4$ to L5. Three patients had a high spinal block (C4, C6 and T1) with mild respiratory and cardiovascular depression 
Table 3 CSF collection

\begin{tabular}{lc}
\hline & Value \\
\hline $\mathrm{N}$ & 413 \\
CSF collected & \\
For general analysis & 408 [99] \\
For storage & $403[98]$ \\
Blood visible in CSF $(\mathrm{n}=412)$ & 17 [4] \\
Time (min) & 2 [2-3], range 1-10 \\
Volume $(\mathrm{mL})$ & Mean 9.3, range 0.1-13.0 \\
$0.1-9.9 \mathrm{~mL}$ & $55[13]$ \\
$\geq 10 \mathrm{~mL}$ & 358 [87]
\end{tabular}

CSF, cerebrospinal fluid. Data are presented as mean $\pm S D$, median [IQR] or frequency [\%].

(hypotension and slight dyspnea), but this was managed supportively without conversion to general anaesthesia. Fifteen patients had an inadequate block. In ten of them, conversion to general anaesthesia was necessary, and in one patient a second spinal attempt was successful. In four patients administration of the potent intravenous opioid analgesic remifentanil was sufficiently effective to enable the operation to be completed.

A vasovagal response with hypotension and bradycardia occurred in $7 \%$ of all patients. Three patients suffered from typical PDPH complaints for several days after discharge. In all cases PDPH resolved spontaneously and no invasive interventions were needed.

\section{CSF and blood collection}

Details of CSF collection are shown in Table 3. The intrathecal space was identified in 425 patients. CSF aspiration was unsuccessful in twelve patients. In the remaining 413 patients, a mean of $9.3 \mathrm{~mL}$ CSF (range, 0.113.0) was obtained. CSF aspiration required a mean (range) of 2 minutes (1-10). In $4 \%$ of cases, blood was clearly visible in the CSF due to a so-called 'traumatic tap'. Blood was collected in 445 (99\%) of the patients.

\section{Medical history, physical examination, cognitive assessment and questionnaires}

A summary of patient medical history, medication use, neurological and cognitive function is provided in Table 4 .
Of the 450 patients, $93 \%$ had at least one co-morbid condition (other than the indication for surgery) and 77\% used medication. A spinal disc herniation was the most common neurological problem. Depression was the most frequent psychiatric disorder and thirty-six patients used antidepressants. A neurological examination and MoCA could be performed for 220 (49\%) and 225 patients (50\%), respectively. MoCA scores ranged from 14 to 30 and $60 \%$ of patients had a normal MoCA score $(\geq 26)$. The different aspects of the neurological examination were normal for $36 \%$ to $85 \%$ of the patients. The results for all 26 -items of the neurological examination can be found in the supplementary appendix online. 101 patients completed the mental health daily diary up to May 2019 (results not presented here). The baseline IDS-SR was completed by 54 patients and the follow-up IDS-SR by 46 patients up to March 2020 (results not presented here).

\section{Routine laboratory analysis}

Results of leucocyte and erythrocyte cell counts, albumin, total protein and glucose concentrations for plasma and CSF are shown in Table 5. The median CSF erythrocyte count was $200 / \mu \mathrm{L}$ and in 70 patients $(17 \%)$ this count exceeded $500 / \mu \mathrm{L}$.

\section{Discussion}

The aim of the ABC is to collect and store CSF from patients undergoing spinal anaesthesia for elective surgery to facilitate future neuroscientific research. We report here our initial findings on the first 450 patients that have been enrolled, resulting in an extensive biobank comprising CSF samples from 413 subjects and plasma samples of 445 subjects. In most patients, $10 \mathrm{~mL}$ CSF could be aspirated as planned. The duration of the spinal anaesthesia procedure was extended by only a few minutes. After CSF aspiration, the responsible anaesthesiologists used typical doses of intrathecal local anaesthetic, and this resulted in sensory block heights consistent with that reported in earlier studies where no CSF was aspirated (43). CSF aspiration did not therefore appear to have influenced the attained block height. Complication rates were lower or comparable with those of previous studies (44-46). Our results show that banking of CSF from a surgical population is feasible and does not appear to pose any significant additional risks to participating patients.

Furthermore, although the project is being conducted 
Table 4 Medical history, medication use and neurological and cognitive function

\begin{tabular}{|c|c|}
\hline & Value \\
\hline $\mathrm{N}$ & 450 \\
\hline Medication use & $347[77]$ \\
\hline Antidepressants & $36[8]$ \\
\hline Medical history & 419 [93] \\
\hline General & $242[54]$ \\
\hline Cardiovascular & $163[36]$ \\
\hline Respiratory & 94 [21] \\
\hline Gastrointestinal & 98 [22] \\
\hline Neurological & $78[17]$ \\
\hline Spinal disc herniation & $22[5]$ \\
\hline CVA/TIA & $15[3]$ \\
\hline Migraine & 7 [2] \\
\hline Meningitis & $4[1]$ \\
\hline Parkinson disease & $4[1]$ \\
\hline Epilepsy & $3[0.7]$ \\
\hline Multiple Sclerosis & $1[0.2]$ \\
\hline Alzheimer disease & $1[0.2]$ \\
\hline Endocrinological & $95[21]$ \\
\hline Urological & 98 [22] \\
\hline Locomotor & $261[58]$ \\
\hline Hematological & $16[4]$ \\
\hline Psychiatric & $36[8]$ \\
\hline MDD & $21[4]$ \\
\hline Anxiety disorder & $10[3]$ \\
\hline Alcohol/drug abuse & 7 [2] \\
\hline ADHD & $4[1]$ \\
\hline Suicide attempt & $2[0.3]$ \\
\hline Psychosis & $2[0.4]$ \\
\hline MoCA score $(n=225)$ & 26 [24-28]; range 14-30 \\
\hline Neurological exam $(n=220)$ & Normal: \\
\hline Cranial nerve function & $168[76]$ \\
\hline Motor function & $154[70]$ \\
\hline Sensory function & $128[58]$ \\
\hline Coordination & $187[85]$ \\
\hline Gait & 154 [70] \\
\hline Reflexes & 79 [36] \\
\hline
\end{tabular}

Data are presented as median [IQR] for MoCA score or number of patients [\%] for medication use, medical history and neurological examination. CVA, cerebro vascular accident; TIA, transient ischemic attack; MDD, major depressive disorder; $\mathrm{ADHD}$, attention deficit hyperactivity disorder; MoCA, Montreal Cognitive Assessment.
Table 5 Plasma and CSF laboratory analysis

\begin{tabular}{lccc}
\hline Material/assay & $\mathrm{n}$ & Median [IQR] & Range \\
\hline Plasma & & & \\
Leucocyte count $\left(\times 10^{9} / \mathrm{L}\right)$ & 439 & $6.4[5.4-7.8]$ & $2.9-35.7$ \\
Albumin (g/L) & 442 & $44[42-47]$ & $20-56$ \\
Total protein (g/L) & 442 & $72[69-76]$ & $33-97$ \\
Glucose (mmol/L) & 437 & $5.4[5.0-5.9]$ & $3.7-17.2$ \\
CSF & & & \\
Leucocyte count $\left(\times 10^{6} / \mathrm{L}\right)$ & 407 & $1.0[1.0-2.0]$ & $0-59$ \\
Erythrocyte count $\left(\times 10^{6} / \mathrm{L}\right)$ & 407 & $200[100-400]$ & $0-32,000$ \\
Albumin (g/L) & 407 & $0.24[0.18-0.32]$ & $0.06-1.00$ \\
Total protein (g/L) & 408 & $0.38[0.30-0.49]$ & $0.14-1.45$ \\
Glucose (mmol/L) & 408 & $3.4[3.2-3.6]$ & $1.1-9.1$ \\
\hline CSF cerebrospinal fluid & & &
\end{tabular}

at a tertiary university hospital, our sample represents a relatively healthy surgical population and a broad selection from the general population. Half of the patients were classified as ASA 1, were discharged the same day of surgery, and the majority of them had only minor comorbidities. On the whole participants were also reasonably cognitively normal, with $60 \%$ of them having MoCA scores in the normal range, which is comparable with the general population (47). The results of the routine laboratory analyses further confirm our view that the patient sample is representative of the general population. Routine analyses showed concentrations within the reference ranges of the UMCG laboratory for plasma leucocyte count, albumin, total protein and glucose and CSF leucocyte count, total protein and glucose. CSF albumin concentrations were consistent with those reported in a study with over 1,000 patients (48).

The $\mathrm{ABC}$ has several strengths. First, the CSF is obtained from relatively healthy patients who require an LP for clinical reasons. Second, the study is embedded in the anaesthesiology department and most patients approached are willing to participate, which helps to generate a sizable sample. Third, the procedures for withdrawal and storage of CSF are highly standardized, providing excellent conditions for future analysis. For instance, sampling CSF in fractions enables investigation of a possible rostrocaudal concentration gradient (16). Furthermore, the routine analysis on the first $2 \mathrm{~mL}$ CSF supports further analysis (49), for example erythrocyte count. CSF does not normally 
contain erythrocytes. In $17 \%$ of the included patients the CSF erythrocyte count exceeded the limit of $500 / \mu \mathrm{L}$, stated as the cut-off value above which assays for biomarkers with high serum concentrations are deemed unreliable (16). This proportion is consistent with previous literature stating that in $14-20 \%$ of patients undergoing a LP a traumatic puncture occurs, causing blood contamination of the CSF (50). Also, additives to some of the stored aliquots stabilize labile analytes like neurotransmitters. In addition, long-duration storage at -80 degrees has been proved to be safe for several biomarkers, and the use of multiple small aliquots will help to avoid repeated freeze/thaw cycles (49,51). Moreover, every patient fasted overnight before the LP, decreasing the effect of diet on laboratory analyses. Also, medication use that can affect CSF and blood biochemistry is registered. An additional strength is the fact that matched plasma and CSF samples are acquired. CSF sampling is more invasive than sampling blood. Our material enables studies of the correlations between CSF- and blood-based biomarker levels, which will indicate whether CSF analyses have added value when compared with plasma analyses. Blood-based biomarkers allow repeated sampling and thus more precise monitoring of disease progression (9). Finally, the biobank has clear phenotyping of patients in terms of their neurocognitive functioning and mental health.

As with every study, the $\mathrm{ABC}$ also has several limitations. First, the sample is selective and consists of patients undergoing spinal anaesthesia. In prior CSF biomarker studies, definitions used for control groups differ and consequently the groups are very heterogeneous. The interpretation of the conclusions of these studies depend on the type of control group and stage of biomarker validation. In general, healthy controls are needed for biomarker discovery studies and in the early stage of marker validation, as these studies usually include a small number of patients and a large contrast between patients and controls is useful. It has been argued that symptomatic controls are relevant for diagnostic markers because they can help to differentiate between the disease of interest and related diseases (9). Fortunately, our neuropsychiatric phenotyping and large sample size allows selection of patients suitable for a particular hypothesis. Second, CSF is collected at only one time point, although biochemical changes in CNS diseases are known not to be static but to fluctuate (52), or follow a circadian rhythm (53). Third, most phenotyping is based on self-report questionnaires, which can be sensitive to response or recall bias. Lastly, possibilities to investigate the associations of CSF biochemistry with neurologic and/or psychiatric diseases in the $\mathrm{ABC}$ data alone will be limited because of the limited number of patients with these disorders in the ABC sample [the proportions of patients in our sample are slightly lower than those described in epidemiological surveys $(54,55)]$. Still, thorough multidimensional psychiatric phenotyping was carried out, enabling investigation of associations between biomarkers and severity of mental health symptoms, ranging from healthy to mental disorder.

\section{Conclusions}

We have described the development of the ABC, the biomaterial sampling and storing methods, the characteristics of the population of patients from whom the material is derived, and our initial procedural and laboratory results. By this means we have shown the feasibility of CSF biobanking, which may pose an example for other researchers. Our large sample of relatively healthy patients, standardized sampling methods as well as the extensive patient phenotyping are unique. This enables analysis of CSF biochemistry to determine healthy-control reference values and to identify novel biomarkers for neuropsychiatric diseases. So far, analyses of neurotransmitters and steroids have started and a study on a possible CSF rostrocaudal gradient is underway. Furthermore, we will focus on detecting biomarkers for depression. We remain open to new collaborations and/or suggestions to extend our study, as a large part of the biomaterial is still stored and available for future analysis.

\section{Acknowledgments}

We extend our appreciation to all patients who participated in this study. The authors would like to thank Berber Mollinga, BSc; David Franken, MD; Sjoukje Karsten, MD; Remco Zielstra, MD; Rajaie Almatrood, BSc; Chris Freeman, MD; Lasse Schopmeyer, MD Willemien Muller, BSc; Myrthe Smit, BSc; and Henk Braam, R.N. (all University of Groningen/University Medical Center Groningen, The Netherlands), for their help during the data collection. The authors wish to thank the involved researchers from the Anesthesiology, Clinical Chemistry, Neurology and Psychiatry departments of the UMCG for their support and contribution to the study concept, design, and data/material collection and analysis. Finally, the authors thank the anaesthesiologists for their assistance with 
the biomaterial collection.

Funding: This study was supported by internal financial resources of the Anesthesiology and Clinical Chemistry departments of the University Medical Center Groningen, The Netherlands.

\section{Footnote}

Data Sharing Statement: Available at http://dx.doi. org/10.21037/atm-20-4498

Reporting Checklist: The authors have completed the STROBE reporting checklist. Available at http://dx.doi. org/10.21037/atm-20-4498

Peer Review File: Available at http://dx.doi.org/10.21037/ atm-20-4498

Conflicts of Interest: All authors have completed the ICMJE uniform disclosure form (available at http://dx.doi. org/10.21037/atm-20-4498). Dr. ARA reports grants and personal fees from Carefusion/BD, personal fees from Terumo, other fees from Rigel Pharma, personal fees from Janssen Pharma, personal fees and other fees from The Medicines Company, personal fees from Ever Pharma, personal fees from PAION, grants and personal fees from Philips, outside the submitted work; all monies received were paid to the author's institution. The other authors have no conflicts of interest to declare.

Ethical Statement: The authors are accountable for all aspects of the work in ensuring that questions related to the accuracy or integrity of any part of the work are appropriately investigated and resolved. The protocol for this research project has been approved by the Medical Ethical Committee of the University Medical Center Groningen (registration number 2016-174) and it conforms to the provisions of the Helsinki Declaration as revised in 2013. All participants signed an informed consent form.

Open Access Statement: This is an Open Access article distributed in accordance with the Creative Commons Attribution-NonCommercial-NoDerivs 4.0 International License (CC BY-NC-ND 4.0), which permits the noncommercial replication and distribution of the article with the strict proviso that no changes or edits are made and the original work is properly cited (including links to both the formal publication through the relevant DOI and the license).
See: https://creativecommons.org/licenses/by-nc-nd/4.0/.

\section{References}

1. Collins PY, Patel V, Joestl SS, et al. Grand challenges in global mental health. Nature 2011;475:27-30.

2. Steel Z, Marnane C, Iranpour C, et al. The global prevalence of common mental disorders: a systematic review and meta-analysis 1980-2013. Int J Epidemiol 2014;43:476-93.

3. GBD 2016 Neurology Collaborators. Global, regional, and national burden of neurological disorders, 1990-2016: a systematic analysis for the Global Burden of Disease Study 2016. Lancet Neurol 2019;18:459-80.

4. Kendell R, Jablensky A. Distinguishing between the validity and utility of psychiatric diagnoses. Am J Psychiatry 2003;160:4-12.

5. Ryan J, Fransquet P, Wrigglesworth J, et al. Phenotypic Heterogeneity in Dementia: A Challenge for Epidemiology and Biomarker Studies. Front Public Health 2018;6:181.

6. Jack CR Jr, Bennett DA, Blennow K, et al. NIA-AA Research Framework: Toward a biological definition of Alzheimer's disease. Alzheimers Dement 2018;14:535-62.

7. Singh I, Rose N. Biomarkers in psychiatry. Nature 2009;460:202-7.

8. Lewczuk P, Riederer P, O'Bryant SE, et al. Cerebrospinal fluid and blood biomarkers for neurodegenerative dementias: An update of the Consensus of the Task Force on Biological Markers in Psychiatry of the World Federation of Societies of Biological Psychiatry. World J Biol Psychiatry 2018;19:244-328.

9. Teunissen CE, Tumani H, Engelborghs S, et al. Biobanking of CSF: international standardization to optimize biomarker development. Clin Biochem 2014;47:288-92.

10. Sühs KW, Novoselova N, Kuhn M, et al. Kynurenine Is a Cerebrospinal Fluid Biomarker for Bacterial and Viral Central Nervous System Infections. J Infect Dis 2019;220:127-38.

11. Parnetti L, Gaetani L, Eusebi P, et al. CSF and blood biomarkers for Parkinson's disease. Lancet Neurol 2019;18:573-86.

12. Tumani H, Hartung HP, Hemmer B, et al. Cerebrospinal fluid biomarkers in multiple sclerosis. Neurobiol Dis 2009;35:117-27.

13. Janssens J, Vermeiren Y, van Faassen M, et al. Monoaminergic and Kynurenergic Characterization of 
Frontotemporal Dementia and Amyotrophic Lateral Sclerosis in Cerebrospinal Fluid and Serum. Neurochem Res 2020;45:1191-201.

14. Engelborghs S, Niemantsverdriet E, Struyfs H, et al. Consensus guidelines for lumbar puncture in patients with neurological diseases. Alzheimers Dement (Amst) 2017;8:111-26.

15. Doherty CM, Forbes RB. Diagnostic Lumbar Puncture. Ulster Med J 2014;83:93-102.

16. Teunissen CE, Petzold A, Bennett JL, et al. A consensus protocol for the standardization of cerebrospinal fluid collection and biobanking. Neurology 2009;73:1914-22.

17. Bielekova B, Martin R. Development of biomarkers in multiple sclerosis. Brain 2004;127:1463-78.

18. van der Vuurst de Vries RM, Mescheriakova JY, Runia TF, et al. Soluble CD27 Levels in Cerebrospinal Fluid as a Prognostic Biomarker in Clinically Isolated Syndrome. JAMA Neurol 2017;74:286-92.

19. Beijers L, Wardenaar KJ, Bosker FJ, et al. Biomarkerbased subtyping of depression and anxiety disorders using Latent Class Analysis. A NESDA study. Psychol Med 2019;49:617-27.

20. Scarr E, Millan MJ, Bahn S, et al. Biomarkers for Psychiatry: The Journey from Fantasy to Fact, a Report of the 2013 CINP Think Tank. Int J Neuropsychopharmacol 2015;18:pyv042.

21. Pech J, Forman J, Kessing LV, et al. Poor evidence for putative abnormalities in cerebrospinal fluid neurotransmitters in patients with depression versus healthy non-psychiatric individuals: A systematic review and meta-analyses of 23 studies. J Affect Disord 2018;240:6-16.

22. Ruhé HG, Mason NS, Schene AH. Mood is indirectly related to serotonin, norepinephrine and dopamine levels in humans: a meta-analysis of monoamine depletion studies. Mol Psychiatry 2007;12:331-59.

23. Aalten P, Ramakers IH, Biessels GJ, et al. The Dutch Parelsnoer Institute--Neurodegenerative diseases; methods, design and baseline results. BMC Neurol 2014;14:254.

24. Komori M, Blake A, Greenwood M, et al. Cerebrospinal fluid markers reveal intrathecal inflammation in progressive multiple sclerosis. Ann Neurol 2015;78:3-20.

25. Luykx JJ, Bakker SC, Lentjes E, et al. Season of sampling and season of birth influence serotonin metabolite levels in human cerebrospinal fluid. PLoS One 2012;7:e30497.

26. González-Quevedo A, García JC, Fernández R, et al. Monoamine metabolites in normal human cerebrospinal fluid and in degenerative diseases of the central nervous system. Bol Estud Med Biol 1993;41:13-9.

27. Dhondt JL. Difficulties in establishing reference intervals for special fluids: the example of 5-hydroxyindoleacetic acid and homovanillic acid in cerebrospinal fluid. Clin Chem Lab Med 2004;42:833-41.

28. Hyland K, Surtees RA, Heales SJ, et al. Cerebrospinal fluid concentrations of pterins and metabolites of serotonin and dopamine in a pediatric reference population. Pediatr Res 1993;34:10-4.

29. Hartikainen P, Soininen H, Reinikainen KJ, et al. Neurotransmitter markers in the cerebrospinal fluid of normal subjects. Effects of aging and other confounding factors. J Neural Transm Gen Sect 1991;84:103-17.

30. Eklundh T, Eriksson M, Sjoberg S, et al. Monoamine precursors, transmitters and metabolites in cerebrospinal fluid: a prospective study in healthy male subjects. J Psychiatr Res 1996;30:201-8.

31. Evans RW. Complications of lumbar puncture. Neurol Clin 1998;16:83-105.

32. Kapur S, Phillips AG, Insel TR. Why has it taken so long for biological psychiatry to develop clinical tests and what to do about it? Mol Psychiatry 2012;17:1174-9.

33. Reijs BL, Teunissen CE, Goncharenko N, et al. The Central Biobank and Virtual Biobank of BIOMARKAPD: A Resource for Studies on Neurodegenerative Diseases. Front Neurol 2015;6:216.

34. Kang JH, Korecka M, Figurski MJ, et al. The Alzheimer's Disease Neuroimaging Initiative 2 Biomarker Core: A review of progress and plans. Alzheimers Dement 2015;11:772-91.

35. Toledo JB, Van Deerlin VM, Lee EB, et al. A platform for discovery: The University of Pennsylvania Integrated Neurodegenerative Disease Biobank. Alzheimers Dement 2014;10:477-484.e1.

36. Myhr KM, Grytten N, Aarseth JH, et al. The Norwegian Multiple Sclerosis National Competence Centre and National Multiple Sclerosis registry -- a resource for clinical practice and research. Acta Neurol Scand Suppl 2006;183:37-40.

37. Mulugeta A, Zhou A, King C, et al. Association between major depressive disorder and multiple disease outcomes: a phenome-wide Mendelian randomisation study in the UK Biobank. Mol Psychiatry 2020;25:1469-76.

38. Kuzman MR, Medved V, Terzic J, et al. Genomewide expression analysis of peripheral blood identifies candidate biomarkers for schizophrenia. J Psychiatr Res 2009;43:1073-7. 


\section{Page 12 of 12}

39. Monserrate AE, Ryman DC, Ma S, et al. Factors associated with the onset and persistence of post-lumbar puncture headache. JAMA Neurol 2015;72:325-32.

40. Nasreddine ZS, Phillips NA, Bedirian V, et al. The Montreal Cognitive Assessment, MoCA: a brief screening tool for mild cognitive impairment. J Am Geriatr Soc 2005;53:695-9.

41. Kroenke K, Spitzer RL, Williams JB, et al. The Patient Health Questionnaire Somatic, Anxiety, and Depressive Symptom Scales: a systematic review. Gen Hosp Psychiatry 2010;32:345-59.

42. Rush AJ, Gullion CM, Basco MR, et al. The Inventory of Depressive Symptomatology (IDS): psychometric properties. Psychol Med 1996;26:477-86.

43. Pitkänen M, Haapaniemi L, Tuominen M, et al. Influence of age on spinal anaesthesia with isobaric $0.5 \%$ bupivacaine. Br J Anaesth 1984;56:279-84.

44. Zorrilla-Vaca A, Mathur V, Wu CL, et al. The Impact of Spinal Needle Selection on Postdural Puncture Headache: A Meta-Analysis and Metaregression of Randomized Studies. Reg Anesth Pain Med 2018;43:502-8.

45. Aasvang EK, Laursen MB, Madsen J, et al. Incidence and related factors for intraoperative failed spinal anaesthesia for lower limb arthroplasty. Acta Anaesthesiol Scand 2018;62:993-1000.

46. Uppal V, Retter S, Shanthanna H, et al. Hyperbaric Versus Isobaric Bupivacaine for Spinal Anesthesia: Systematic Review and Meta-analysis for Adult Patients Undergoing Noncesarean Delivery Surgery. Anesth Analg 2017;125:1627-37.

47. Thomann AE, Goettel N, Monsch RJ, et al. The Montreal Cognitive Assessment: Normative Data from a German-

Cite this article as: Tigchelaar C, Atmosoerodjo SD, van Faassen M, Wardenaar KJ, De Deyn PP, Schoevers RA, Kema IP, Absalom AR. The anaesthetic biobank of cerebrospinal fluid: a unique repository for neuroscientific biomarker research. Ann Transl Med 2021;9(6):455. doi: 10.21037/atm-20-4498
Tigchelaar et al. The Anaesthetic Biobank of Cerebrospinal fluid

Speaking Cohort and Comparison with International Normative Samples. J Alzheimers Dis 2018;64:643-55.

48. Seyfert S, Faulstich A, Marx P. What determines the CSF concentrations of albumin and plasma-derived IgG? J Neurol Sci 2004;219:31-3.

49. Bruegel M, Planert M, Baumann S, et al. Standardized peptidome profiling of human cerebrospinal fluid by magnetic bead separation and matrix-assisted laser desorption/ionization time-of-flight mass spectrometry. J Proteomics 2009;72:608-15.

50. Petzold A, Sharpe LT, Keir G. Spectrophotometry for cerebrospinal fluid pigment analysis. Neurocrit Care 2006;4:153-62.

51. Willemse EAJ, van Uffelen KWJ, van der Flier WM, et al. Effect of long-term storage in biobanks on cerebrospinal fluid biomarker Abeta1-42, T-tau, and P-tau values. Alzheimers Dement (Amst) 2017;8:45-50.

52. Bateman RJ, Wen G, Morris JC, et al. Fluctuations of CSF amyloid-beta levels: implications for a diagnostic and therapeutic biomarker. Neurology 2007;68:666-9.

53. Chung S, Son GH, Kim K. Circadian rhythm of adrenal glucocorticoid: its regulation and clinical implications. Biochim Biophys Acta 2011;1812:581-91.

54. Bandelow B, Michaelis S. Epidemiology of anxiety disorders in the 21st century. Dialogues Clin Neurosci 2015;17:327-35.

55. Kessler RC, Berglund P, Demler O, et al. Lifetime prevalence and age-of-onset distributions of DSM-IV disorders in the National Comorbidity Survey Replication. Arch Gen Psychiatry 2005;62:593-602. Erratum in: Arch Gen Psychiatry 2005;62:768. 


\begin{tabular}{|c|c|c|c|c|c|c|}
\hline Neurological assessment & \multicolumn{2}{|c|}{ Normal } & \multicolumn{2}{|c|}{ Abnormal } & \multicolumn{2}{|c|}{ Not tested } \\
\hline Cranial nerve function & \multicolumn{2}{|c|}{168} & \multirow{2}{*}{\multicolumn{2}{|c|}{11}} & \multirow{2}{*}{\multicolumn{2}{|c|}{0}} \\
\hline Visual field & \multicolumn{2}{|c|}{209} & & & & \\
\hline Eye inspection & \multicolumn{2}{|c|}{211} & \multicolumn{2}{|c|}{9} & \multicolumn{2}{|c|}{0} \\
\hline Pupil reflexes & \multicolumn{2}{|c|}{216} & \multicolumn{2}{|c|}{4} & \multicolumn{2}{|c|}{0} \\
\hline Eye movement & \multicolumn{2}{|c|}{210} & \multicolumn{2}{|c|}{9} & \multicolumn{2}{|c|}{1} \\
\hline Hearing & & 84 & \multicolumn{2}{|c|}{36} & \multicolumn{2}{|c|}{0} \\
\hline Facial sensation & \multicolumn{2}{|c|}{219} & \multicolumn{2}{|c|}{1} & \multicolumn{2}{|c|}{0} \\
\hline Facial strength & \multicolumn{2}{|c|}{220} & \multicolumn{2}{|c|}{0} & \multicolumn{2}{|c|}{0} \\
\hline Facial expressions & & 18 & & 2 & & \\
\hline Palatal movement & & 20 & & 0 & & \\
\hline Shoulder/neck strength & & 17 & & 3 & & \\
\hline Tongue movement/strength & & 19 & & 1 & & \\
\hline Motor function & & 154 & & & & \\
\hline Upper extremity strength & & 204 & & 6 & & \\
\hline Lower extremity strength & & 60 & & 9 & & \\
\hline Pronator drift & & 16 & & 3 & & \\
\hline Sensory function & & 28 & & & & \\
\hline Sensation in extremities & & 32 & & 8 & & \\
\hline Romberg & & 204 & & 1 & & \\
\hline Coordination & & 87 & & & & \\
\hline Finger tapping & & 10 & & 9 & & \\
\hline Rapid alternating movement & & 209 & & 0 & & \\
\hline Finger-nose test & & 14 & & 6 & & \\
\hline Heel-shin test & & 204 & & 2 & & \\
\hline Gait & & 154 & & 9 & & \\
\hline Reflexes & & 79 & & & & \\
\hline Reflexes & Absent & Reduced & Normal & Increased & Clonus & Not tested \\
\hline Biceps & & & & & & \\
\hline Right & 19 & 9 & 189 & 1 & 0 & 2 \\
\hline Left & 19 & 10 & 188 & 1 & 0 & 2 \\
\hline Triceps & & & & & & \\
\hline Right & 63 & 21 & 132 & 1 & 0 & 3 \\
\hline Left & 62 & 19 & 136 & 1 & 0 & 2 \\
\hline Knee & & & & & & \\
\hline Right & 26 & 17 & 166 & 5 & 0 & 6 \\
\hline Left & 26 & 13 & 172 & 7 & 0 & 2 \\
\hline Heel & & & & & & \\
\hline Right & 61 & 15 & 137 & 2 & 0 & 5 \\
\hline Left & 61 & 14 & 142 & 2 & 0 & 1 \\
\hline Plantar & Indiffer & Abr & nal & Normal & Not & sted \\
\hline Right & 74 & & & 134 & & \\
\hline Left & 74 & & & 138 & & \\
\hline
\end{tabular}

Figure S1 Neurological examination ( $\mathrm{n}=220)$. Data are number of patients. For the main aspects (cranial nerve function, motor function, sensory function, coordination, gait and reflexes), number of patients with normal results for all items for that area are listed. 